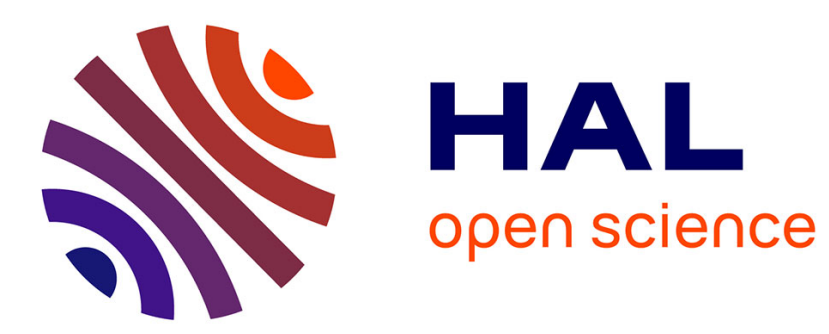

\title{
Recursive equations for the predictive distributions of some determinantal processes
}

\author{
D.M. Cifarelli, S. Fortini
}

\section{To cite this version:}

D.M. Cifarelli, S. Fortini. Recursive equations for the predictive distributions of some determinantal processes. Statistics and Probability Letters, 2010, 81 (1), pp.8. 10.1016/j.spl.2010.09.012 . hal00698868

\section{HAL Id: hal-00698868 \\ https://hal.science/hal-00698868}

Submitted on 18 May 2012

HAL is a multi-disciplinary open access archive for the deposit and dissemination of scientific research documents, whether they are published or not. The documents may come from teaching and research institutions in France or abroad, or from public or private research centers.
L'archive ouverte pluridisciplinaire HAL, est destinée au dépôt et à la diffusion de documents scientifiques de niveau recherche, publiés ou non, émanant des établissements d'enseignement et de recherche français ou étrangers, des laboratoires publics ou privés. 


\section{Accepted Manuscript}

Recursive equations for the predictive distributions of some

determinantal processes

D.M. Cifarelli, S. Fortini

PII:

S0167-7152(10)00262-2

DOI:

10.1016/j.spl.2010.09.012

Reference: $\quad$ STAPRO 5798

To appear in: Statistics and Probability Letters

Received date: 14 July 2010

Revised date: 14 September 2010

Accepted date: 14 September 2010

Please cite this article as: Cifarelli, D.M., Fortini, S., Recursive equations for the predictive distributions of some determinantal processes. Statistics and Probability Letters (2010), doi:10.1016/j.spl.2010.09.012

This is a PDF file of an unedited manuscript that has been accepted for publication. As a service to our customers we are providing this early version of the manuscript. The manuscript will undergo copyediting, typesetting, and review of the resulting proof before it is published in its final form. Please note that during the production process errors may be discovered which could affect the content, and all legal disclaimers that apply to the journal pertain. 


\title{
Recursive equations for the predictive distributions of some determinantal processes
}

\author{
D. M. Cifarelli, S. Fortini \\ Bocconi University
}

\begin{abstract}
The paper provides recursive equations for the predictive distributions of one-dependent and two-dependent determinantal processes. Fixed order recursive equations can be applied both to efficiently simulate trajectories and to explore properties of the process.
\end{abstract}

Keywords: Determinantal processes; predictive distributions; m-dependence; negative association 2000 MSC: 60G10; 60G25

\section{Introduction}

Determinantal processes have been studied by many authors, mainly in the framework of fermion point processes and fields, and applied in different contexts, including physics, random matrix theory, representation theory, and ergodic theory (see Lyons, 2003, Lyons and Steif, 2003, Shirai and Takahashi, 2003 and the references therein). In words, a determinantal process is a $\{0,1\}$-valued process, indexed by a finite or countable set, whose cylinder probabilities are determinants.

A recent delightful paper on one-dependent determinantal processes is Borodin et al. (2009). Besides many examples from combinatorics to graph theory, it contains the important result that any one-dependent point process on the integers is determinantal.

Determinantal processes are interesting because they allow one to model conditional negative association (see Lyons, 2003, Lyons and Steif, 2003 and

Email address: sandra.fortini@unibocconi.it (S. Fortini)

${ }^{1}$ Department of Decision Sciences, Via Röntgen 1, 20136 Milano, Italy 
Shirai and Takahashi, 2003 for the precise definition and proofs). In practice, a "one" at a certain site decreases the probability of further "ones" in the process, whatever the values of the process at different sites. For this reason, determinantal processes are used in statistical physics to represent systems of repulsive particles. Stationary determinantal processes (SDP) have interesting properties, such as mixing, entropy positivity, Bernoulli shift, and Gibbs properties (see Lyons and Steif, 2003 and Shirai and Takahashi, 2003).

The present paper focuses on finitely dependent SDP's. In particular, the problem of computing the predictive distributions of one-dependent and two-dependent SDP's is treated.

Finitely dependent SDP's can be used to model local negative interactions between random variables that propagate through the stochastic sequence. In fact, if $\left(X_{n}\right)_{n \in \mathbb{Z}}$ is $m$-dependent, $X_{n}$ and $X_{j}$ are independent when $n<j-m$. Nevertheless, they are not conditionally independent given $X_{n+1}, \ldots, X_{j-1}$. This feature is in some sense the opposite of the Markov property.

Predictive distributions of SDP's are cumbersome. In fact, no simple sufficient statistics can be identified. On the other hand, it is proved in the present paper that recursive equations of fixed order exist for the predictive distributions of one-dependent and two-dependent SDP's.

This paper is organized as follows. In Section 2 we recall the precise definition of an SDP and discuss its parameters. In Section 3 we provide recursive equations for the predictive distributions of one-dependent SDP's and give a characterization of the process in terms of predictive covariances. Some of these results are contained in the technical report Cifarelli and Fortini (2005). The two-dependent case is treated in Section 4. There recursive equations are given, separately, for complex and real parameters. Equations of lower degree hold when the parameters are real. The results are applied to explore properties of the predictive probabilities and to suggest efficient simulation procedures.

\section{Definitions and preliminary results}

Let $E$ be a finite or countable set and let $K$ be an $E \times E$ matrix of complex numbers satisfying $K_{j, m}=\bar{K}_{m, j}$ for every $j, m \in E$ and $\sum_{j, m \in E} K_{j, m} u_{j} \bar{u}_{m} \in$ $[0,1]$ for every $u$ in the complex Hilbert space $l^{2}(E)$. For every finite $E_{1} \subseteq E$ let $K \mid E_{1}$ denote the submatrix of $K$ whose rows and columns are indexed by $E_{1}$. It is possible to define a probability measure $P^{K}$ on the cylinder 
sigma-algebra of $\{0,1\}^{E}$ such that

$$
P^{K}\left\{x \in\{0,1\}^{E}: x_{e}=1, e \in E_{1}\right\}=\operatorname{det}\left(K \mid E_{1}\right)
$$

$\left(E_{1} \subseteq E, E_{1}\right.$ finite) (see Lyons, 2003, Lyons and Steif, 2003 and Shirai and Takahashi, 2003).

Definition 1. A probability measure $P^{K}$ on on the cylinder sigma-algebra of $\{0,1\}^{E}$ satisfying (1) is called a determinantal probability measure. The coordinate process $\left(X_{n}\right)_{n \in \mathbb{Z}}$ of $P^{K}$ is called a determinantal process.

It follows from (1) that for every finite, disjoint $E_{1}, E_{2} \subseteq E$,

$$
P^{K}\left(\left\{x \in\{0,1\}^{E}: x_{e_{1}}=0, e_{1} \in E_{1}, x_{e_{2}}=1, e_{2} \in E_{2}\right\}\right)=\operatorname{det}\left(K^{E_{1}} \mid E_{1} \cup E_{2}\right),
$$

where, for every $E_{1} \subseteq E, K^{E_{1}}$ denotes the matrix that coincides with $K$ except on the rows indexed by $E_{1}$, where it coincides with $I-K$ (see Lyons, 2003 or Shirai and Takahashi, 2003). It follows from (2) that $\left(1-X_{e}\right)_{e \in E}$ has probability distribution $P^{I-K}$ if $\left(X_{e}\right)_{e \in E}$ has probability distribution $P^{K}$. In the following we will refer to this property as "duality".

This paper deals with determinantal probability measures on the cylinder sigma-algebra of $\{0,1\}^{\mathbb{Z}}$. Furthermore attention is restricted to matrices $K$ satisfying $K_{j, m}=k(j-m)$ for some function $k: \mathbb{Z} \rightarrow \mathbb{C}$. Under this condition $P^{K}$ is stationary. In fact $K\left|\left(E_{1}+n\right)=K\right| E_{1}$ for every $E_{1} \subseteq \mathbb{Z}$ and every $n \in \mathbb{Z}$. It is proved in Lyons and Steif (2003) and Shirai and Takahashi $(2003)$ that $k(j)(j \in \mathbb{Z})$ is the $j$-th Fourier coefficient $\hat{f}_{j}$ of a $[0,1]$-valued Lebesgue-measurable function $f$ on the torus $\mathbb{T}=\mathbb{R} / \mathbb{Z}$.

In the following we will write $P^{f}$ instead of $P^{K}$ to stress the dependence on $f$. Furthermore, we will assume that $f$ is neither a.s. equal to one nor a.s. equal to zero. This last condition ensures that $P^{f}$ has full support (see Lyons and Steif, 2003).

Lemma 1. Let $f: \mathbb{T} \rightarrow[0,1]$ be a Lebesgue-measurable function. If $g(s)=$ $f(s+t)$ for some $t \in \mathbb{T}$, then $P^{g}=P^{f}$.

Proof. Let $K_{f}$ and $K_{g}$ be the infinite dimensional matrices whose $(j, m)$-th entry is $\hat{f}_{m-j}$ and $\hat{g}_{m-j}$, respectively $(j, m \in \mathbb{Z})$. Since $\hat{g}_{j}=\exp (i 2 \pi j t) \hat{f}_{j}$ for $j \in \mathbb{Z}$, then $K_{g}=\left(D^{-1} K_{f} D\right)$, where $D$ is the infinite dimensional diagonal matrix whose $(j, j)$-th entry is $\exp (i 2 \pi j t)$. For every finite $A \subseteq \mathbb{Z}$, we have that $\operatorname{det}\left(K_{g} \mid A\right)=\operatorname{det}\left(D^{-1} K_{f} D \mid A\right)=\operatorname{det}\left(D^{-1} \mid A\right) \operatorname{det}\left(K_{f} \mid A\right) \operatorname{det}(D \mid A)=$ $\operatorname{det}\left(K_{f} \mid A\right)$. 
According to the above lemma, there is some degree of arbitrariness in parameterizing an SDP. In particular, one of the $k_{j}$ 's can be taken to be real positive, without loss of generality.

Let us now introduce cylinder probabilities, predictive distributions and predictive probabilities. For every sequence $\left(x_{n}\right)_{n \in \mathbb{Z}}$, let $x_{(n)}=\left(x_{1}, x_{2}, \ldots, x_{n}\right)$ $(n \in \mathbb{N})$. Analogously, let $1_{(n)}$ and $0_{(n)}$ denote the vectors of $n$ ones and zeroes, respectively $(n \geq 1)$. The probability measures $P_{n}^{f}$ defined by

$$
P_{n}^{f}(A)=P^{f}\left(\left\{x \in\{0,1\}^{\mathbb{Z}}: x_{(n)} \in A\right\}\right)
$$

( $n=1,2, \ldots, A \subseteq\{0,1\}^{n}$ ) will be called the cylinder probabilities of $P^{f}$ or of its coordinate process $\left(X_{n}\right)_{n \in \mathbb{Z}}$. Since $P^{f}$ has full support, $P_{n}^{f}(A)>0$ for every non-empty $A \subseteq\{0,1\}^{n}$ and every $n \in \mathbb{N}$. The predictive distributions of $P^{f}$ or of its coordinate process $\left(X_{n}\right)_{n \in \mathbb{Z}}$ are defined by $P^{f}\left(X_{n} \in A \mid X_{(n-1)}=\right.$ $\left.x_{(n-1)}\right)\left(A \subseteq\{0,1\}, n \geq 2, x_{(n-1)} \in\{0,1\}^{n-1}\right)$. Since SDP's are $\{0,1\}$-valued, the predictive distributions are completely characterized by the functions

$$
p_{n}^{f}\left(x_{(n-1)}\right)=P^{f}\left(X_{n}=1 \mid X_{(n-1)}=x_{(n-1)}\right)
$$

$\left(n \geq 2, x_{(n-1)} \in\{0,1\}^{n-1}\right)$. Furthermore, since $P^{f}$ has full support,

$$
p_{n}^{f}\left(x_{(n-1)}\right)=P_{n}^{f}\left(\left\{\left(x_{1}, \ldots, x_{n-1}, 1\right)\right\}\right) / P_{n-1}^{f}\left(\left\{\left(x_{1}, \ldots, x_{n-1}\right)\right\} .\right.
$$

In the following, we will call the $p_{n}^{f}$ the predictive probabilities of $P^{f}$ or of its coordinate process $\left(X_{n}\right)_{n \in \mathbb{Z}}$.

Finally, let us recall the definition of $m$-dependence.

Definition 2. A random sequence $\left(X_{n}\right)_{n \in \mathbb{Z}}$ (or its probability distribution) is said to be $m$-dependent $(m \in \mathbb{N})$ if $\left(X_{n}\right)_{n<j}$ is stochastically independent of $\left(X_{n}\right)_{n \geq j+m}$ for every $j \in \mathbb{Z}$.

Since for $\{0,1\}$-valued processes zero correlation implies stochastic independence and since, for SDP, $\operatorname{cov}\left(X_{n}, X_{n+j}\right)=-\left|k_{j}\right|^{2}(j=1,2, \ldots),\left(X_{n}\right)_{n \in \mathbb{Z}}$ is $m$-dependent if and only if $k_{j}=0$ for every $j>m(m \in \mathbb{N})$. Hence an SDP is $m$-dependent if and only if $f(s)$ can be written as $\sum_{j=-m}^{m} k_{j} \exp (i 2 \pi j s)$.

\section{One-dependent stationary determinantal processes}

Let $P^{f}$ be a one-dependent SDP such that $f$ is neither a.s. equal to zero nor a.s.equal to one. By the results in the previous section, we can suppose that

$$
f(s)=k_{0}+2 k_{1} \cos (2 \pi s) \quad\left(s \in \mathbb{T}, k_{0} \in(0,1), k_{1} \in\left[0, \min \left(k_{0}, 1-k_{0}\right) / 2\right]\right) .
$$


Lemma 2. Let $P^{f}$ be a stationary determinantal probability measure with $f$ as in (4). For every $x \in\{0,1\}^{\mathbb{Z}}$ and every $n \geq 2$, we have

$P_{n}^{f}\left(\left\{x_{(n)}\right\}\right)=k_{0}^{x_{n}}\left(1-k_{0}\right)^{1-x_{n}} P_{n-1}^{f}\left(\left\{x_{(n-1)}\right\}\right)-(-1)^{x_{n}+x_{n-1}} k_{1}^{2} P_{n-2}^{f}\left(\left\{x_{(n-2)}\right\}\right)$,

where $P_{0}^{f}\left(\left\{x_{(0)}\right\}\right):=1$.

Proof. Let $x_{1}, x_{2}, \ldots$ be a fixed $\{0,1\}$-valued sequence. Define the infinite dimensional matrix $\left[A_{j l}\right]_{j, l=1,2 \ldots}$ by $A_{j l}=k_{0}^{x_{j}}\left(1-k_{0}\right)^{1-x_{j}}$ if $l=j, A_{j l}=$ $(-1)^{x_{j}+1} k_{1}$ if $l=j-1, j+1$, and $A_{j l}=0$ otherwise. Then $P_{n}^{f}\left(\left\{x_{(n)}\right\}\right)=$ $\operatorname{det} A \mid\{1, \ldots, n\}$. Equation (5) is obtained by $\operatorname{developing} \operatorname{det} A \mid\{1, \ldots, n\}$ along the last row and column.

Theorem 1. Let $P^{f}$ be a stationary determinantal probability measure with $f$ as in (4). For every $x \in\{0,1\}^{\mathbb{Z}}$ and every $n \geq 2$, we have

$$
p_{n}^{f}\left(x_{(n-1)}\right)=k_{0}+k_{1}^{2}\left(1-p_{n-1}^{f}\left(x_{(n-2)}\right)\right)^{-1}\left(1-1 / p_{n-1}^{f}\left(x_{(n-2)}\right)\right)^{x_{n-1}}
$$

where $p_{1}^{f}\left(x_{(0)}\right):=P_{1}^{f}(\{1\})$.

Proof. Equation (6) is obtained from Lemma 2 by computing

$$
p_{n}^{f}\left(x_{(n-1)}\right)=P_{n}^{f}\left(\left\{\left(x_{1}, \ldots, x_{n-1}, 1\right)\right\}\right) / P_{n-1}^{f}\left(\left\{\left(x_{1}, \ldots, x_{n-1}\right)\right\}\right)
$$

and observing that

$$
\frac{P_{n-1}^{f}\left(\left\{\left(x_{1}, \ldots, x_{n-1}\right)\right\}\right)}{P_{n-2}^{f}\left(\left\{\left(x_{1}, \ldots, x_{n-2}\right)\right\}\right)}=p_{n-1}^{f}\left(x_{(n-2)}\right)^{x_{n-1}}\left(1-p_{n-1}^{f}\left(x_{(n-2)}\right)\right)^{1-x_{n-1}} .
$$

Example 1. Let $\left(X_{n}\right)_{n \in \mathbb{Z}}$ be a one-dependent SDP with parameter $f$ as in (4). We have that $p:=\lim _{n \rightarrow \infty} p_{n}^{f}\left(1_{(n-1)}\right)=\exp \int_{\mathbb{T}} \log f d \lambda$ (see Lyons and Steif, 2003). Theorem 1 can be used to evaluate $p$ without computing the integral. In fact, by (6), $p$ must satisfy the equation $p^{2}-k_{0} p+k_{1}^{2}=0$. Equation (6) together with $p_{1}^{f}=k_{0}$ implies that $p_{n}^{f}\left(1_{(n-1)}\right) \geq k_{0} / 2$ for every $n$. Hence $p=k_{0} / 2+\sqrt{\left(k_{0} / 2\right)^{2}-k_{1}^{2}}$. By duality, the limit probability of a "one" after an $n$-long sequence of "zeroes" is $q=\left(1+k_{0}\right) / 2-\sqrt{\left(\left(1-k_{0}\right) / 2\right)^{2}-k_{1}^{2}}$.

Depending on the values of $k_{0}$ and $k_{1}, p$ can range between $k_{0} / 2$ (when $k_{0} \leq 1 / 2$ and $k_{1}=k_{0} / 2$ ) and $k_{0}$ (when $k_{1}=0$, i.e. in the i.i.d. case) and $q$ can range between $k_{0}$ and $\left(1+k_{0}\right) / 2$. 
Equation (6), together with $P_{1}^{f}(\{1\})=k_{0}$, characterizes one-dependent SDP's. The next result gives a characterization of SDP's within the class of one-dependent processes.

Corollary 1. Let $\left(X_{n}\right)_{n \in \mathbf{Z}}$ be a $\{0,1\}$-valued, stationary, one-dependent process with probability distribution $P$. A necessary and sufficient condition for $P=P^{f}$ with $f$ as in (4) is

$$
\left\{\begin{array}{l}
E\left(X_{1}\right)=k_{0} \\
\operatorname{Cov}\left(X_{n+1}, X_{n+2} \mid X_{1}, \ldots, X_{n}\right)=-k_{1}^{2} \quad P-a . s . \quad(n \geq 0) .
\end{array}\right.
$$

Proof. Let $\left(P_{n}\right)_{n \in \mathbb{N}}$ and $\left(p_{n}\right)_{n \in \mathbb{N}}$ denote the sequences of the cylinder and predictive probabilities of $P$ respectively. Let us first prove that $(7)$ holds if $P=P^{f}$. We have that $E\left(X_{1}\right)=P^{f}(\{1\})=k_{0}$. Furthermore, equation (6) yields

$$
\begin{aligned}
& \operatorname{Cov}\left(X_{n+1}, X_{n+2} \mid X_{1}=x_{1}, \ldots, X_{n}=x_{n}\right) \\
& =p_{n+1}^{f}\left(x_{1}, \ldots, x_{n}\right) p_{n+2}^{f}\left(x_{1}, \ldots, x_{n}, 1\right)-k_{0} p_{n+1}^{f}\left(x_{1}, \ldots, x_{n}\right)=-k_{1}^{2}
\end{aligned}
$$

for every fixed $\left(x_{1}, x_{2}, \ldots, x_{n}\right) \in\{0,1\}^{n}$.

To prove sufficiency, notice that the distribution of a $\{0,1\}$-valued, stationary, one-dependent process is completely characterized by the sequence $\left(P_{n}\left(\left\{1_{(n)}\right\}\right)\right)_{n \geq 1}$. It follows from $(7)$ that $P_{1}^{f}(\{1\})=E\left(X_{1}\right)=k_{0}=P_{1}^{f}(\{1\})$. Furthermore, by induction, (7) yields $P_{n}\left(\left\{1_{(n)}\right\}\right) \neq 0$ and $P_{n+2}\left(\left\{1_{(n+2)}\right\}\right)-$ $k_{0} P_{n+1}\left(\left\{1_{(n+1)}\right\}\right)=-k_{1}^{2} P_{n}\left(\left\{1_{(n)}\right\}\right)$. By Lemma 2, $P_{n}\left(\left\{1_{(n)}\right\}\right)=P_{n}^{f}\left(\left\{1_{(n)}\right\}\right)$ for every $n \geq 1$.

Example 2. Equation (7) can be exploited to give a new proof that a onedependent $\operatorname{SDP}\left(X_{n}\right)_{n \in Z}$ is a two-block factor (see Broman, 2005). We recall that a process $\left(X_{n}\right)_{n \in Z}$ is an $m$-block factor if there exist a measurable function $h$ of $m$ variables and an i.i.d. process $\left(Y_{n}\right)_{n \in \mathbf{Z}}$ such that $\left(X_{n}\right)_{n \in Z} \stackrel{d}{=}\left(h\left(Y_{n}, Y_{n+1}, \ldots, Y_{n+m-1}\right)\right)_{n \in Z}$, where $\stackrel{d}{=}$ denotes equality in distribution. Let $\left(X_{n}\right)_{n \in Z}$ be an SDP with $f$ as in (4). We can suppose $0<k_{0} \leq 1 / 2$ without loss of generality. In fact, in the degenerate case $k_{0}=0$, the two-block factor condition is obvious; while for $k_{0}>1 / 2$ it can be proved by duality. To prove that $\left(X_{n}\right)_{n \in Z}$ is a two-block factor, consider a sequence $\left(Y_{n}\right)_{n \in \mathbf{Z}}$ of i.i.d. random variables with uniform distribution on $[0,1]$. We will show that $\left(1_{A}\left(Y_{n}, Y_{n-1}\right)\right)_{n \in Z} \stackrel{d}{=}\left(X_{n}\right)_{n \in Z}$ for a suitable choice 
of a Borel set $A \subseteq[0,1]^{2}$. Since, for every $A,\left(1_{A}\left(Y_{n}, Y_{n+1}\right)\right)_{n \in Z}$ is a $\{0,1\}$ valued, stationary, one-dependent process, according to Corollary 1 , it is sufficient to find $A$ such that $E\left(1_{A}\left(Y_{1}, Y_{2}\right)\right)=k_{0}$ and

$$
\operatorname{Cov}\left(1_{A}\left(Y_{n+1}, Y_{n+2}\right), 1_{A}\left(Y_{n+2}, Y_{n+3}\right) \mid 1_{A}\left(Y_{1}, Y_{2}\right), \ldots, 1_{A}\left(Y_{n}, Y_{n+1}\right)\right)=-k_{1}^{2}
$$

for every $n \geq 1$. The first condition is true if $(\lambda \times \lambda)(A)=k_{0}$, where $\lambda$ is the Lebesgue measure on $[0,1]$. A sufficient condition for the second equality is

$$
\begin{array}{r}
E\left(1_{A}\left(Y_{n+1}, Y_{n+2}\right) 1_{A}\left(Y_{n+2}, Y_{n+3}\right) \mid Y_{1}, \ldots, Y_{n+1}\right) \\
=k_{0} E\left(1_{A}\left(Y_{n+1}, Y_{n+2}\right) \mid Y_{1}, \ldots, Y_{n+1}\right)-k_{1}^{2}
\end{array}
$$

a.s. or, equivalently,

$P\left(\left(Y_{n+1}, Y_{n+2}\right) \in A,\left(Y_{n+2}, Y_{n+3}\right) \in A \mid Y_{n+1}\right)=k_{0} P\left(\left(Y_{n+1}, Y_{n+2}\right) \in A \mid Y_{n+1}\right)-k_{1}^{2}$,

a.s. This last equation holds if $A$ satisfies $\int_{A_{y}} \lambda\left(A_{z}\right) \lambda(d z)=k_{0} \lambda\left(A_{y}\right)-k_{1}^{2}$ a.s., where, for every $x, A_{x}=\{y \in[0,1]:(x, y) \in A\}$. An example is $A=A_{1} \cup A_{2} \cup A_{3}$ with $A_{1}=\left[0, k_{0} / 2+\sqrt{k_{0}^{2} / 4-k_{1}^{2}}\right] \times[0,1 / 2], A_{2}=\left[1-k_{0} / 2-\right.$ $\left.\sqrt{k_{0}^{2} / 4-k_{1}^{2}}, 1\right] \times[0,1 / 2]$, and $A_{3}=[0,1] \times\left[1 / 2,1 / 2+k_{0} / 2-\sqrt{k_{0}^{2} / 4-k_{1}^{2}}\right]$.

The next result can be used to sample sequentially from $P^{f}$. The advantage of this procedure, if compared with simulations using determinants, is that the computation complexity is not increasing with $n$.

Corollary 2. Let $\left(Z_{n}\right)_{n \in \mathbb{N}}$ be a sequence of independent random variables defined on a probability space $(\Omega, \mathcal{F}, P)$, uniformly distributed on $[0,1]$. Furthermore, let $X_{n}=1_{\left[0, W_{n}\right]}\left(Z_{n}\right)(n \geq 1), W_{1}=k_{0}$ and

$$
W_{n}=k_{0}+\frac{k_{1}^{2}}{1-W_{n-1}}\left(1-\frac{1}{W_{n-1}}\right)^{X_{n-1}} \quad(n>1),
$$

with $k_{0} \in(0,1)$ and $k_{1} \in\left[0, \min \left(k_{0}, 1-k_{0}\right) / 2\right]$. Then, for every $n \geq 1, X_{(n)}$ has probability distribution $P_{n}^{f}$ with $f(s)=k_{0}+2 k_{1} \cos (2 \pi s)(s \in \mathbb{T})$.

Proof. By induction, (6) implies $W_{n}=p_{n}^{f}\left(X_{(n-1)}\right)$ for every $n \geq 1$. Hence for every $n \geq 1, P\left(X_{n}=1 \mid X_{1}, \ldots, X_{n-1}\right)=W_{n}=p_{n}^{f}\left(X_{(n-1)}\right)$. It follows that for every $\left(x_{1}, x_{2}, \ldots\right) \in\{0,1\}^{\infty}$ and every $n \geq 1, P\left(X_{(n)}=x_{(n)}\right)=P\left(X_{1}=\right.$ $\left.x_{1}\right) \prod_{j=2}^{n} P\left(X_{j}=x_{j} \mid X_{(j-1)}=x_{(j-1)}\right)=k_{0}^{x_{1}}\left(1-k_{0}\right)^{1-x_{1}} \prod_{j=1}^{n} p_{j}^{f}\left(x_{(j-1)}\right)=$ $P_{n}^{f}\left(\left\{x_{(n)}\right\}\right)$ 


\section{Two-dependent stationary determinantal processes}

Let $P^{f}$ be an SDP with

$$
f(s)=\sum_{j=-2}^{2} k_{j} \exp (i 2 \pi j s) \quad(s \in \mathbb{T})
$$

where $k_{-j}=\bar{k}_{j}$. By Lemma 1 , we can assume $k_{-1}=k_{1} \in \mathbb{R}$, without loss of generality.

Lemma 3. Let $P^{f}$ be a stationary, two-dependent determinantal probability measure with $f$ as in $(8), k_{0} \in(0,1), k_{1} \in \mathbb{R}$, and $k_{2} \in \mathbb{C}$. Then, for every $x \in\{0,1\}^{\mathbb{Z}}$ and every $n \geq 3$

$$
\begin{aligned}
P_{n}^{f}\left(\left\{x_{(n)}\right\}\right) & =\sum_{j=2}^{6 \wedge n} a_{j}(-1)^{\sum_{u=0}^{j-1} x_{n-u}} P_{n-j}^{f}\left(\left\{x_{(n-j)}\right\}\right) \\
& +\sum_{j \in\{1,3\}} c_{j}(-1)^{\sum_{u=0}^{j-1} x_{n-u}} b\left(k_{0} ; x_{n-j+1}\right) P_{n-j}^{f}\left(\left\{x_{(n-j)}\right\}\right) \\
& +\sum_{j \in\{3,5\}, j \leq n} c_{j}(-1)^{\sum_{u=0}^{j-1} x_{n-u}} b\left(k_{0} ; x_{n-j+2}\right) P_{n-j}^{f}\left(\left\{x_{(n-j)}\right\}\right)
\end{aligned}
$$

where $P_{0}^{f}\left(\left\{x_{(0)}\right\}\right):=1$,

$$
\begin{gathered}
a_{2}=\left|k_{2}\right|^{2}-k_{1}^{2} \quad a_{3}=-2 k_{1}^{2}\left|k_{2}\right| \cos \theta \quad a_{4}=\left(\left|k_{2}\right|^{2}-k_{1}^{2}\right)\left|k_{2}\right|^{2} \quad a_{5}=0 \quad a_{6}=-\left|k_{2}\right|^{6} \\
b(p ; x)=(-p)^{x}(1-p)^{1-x} \quad(p \in(0,1), x \in\{0,1\}) \\
c_{j}=\left(-\left|k_{2}\right|^{2}\right)^{(j-1) / 2} \quad(j=1,3,5)
\end{gathered}
$$

Proof. Let $x_{1}, x_{2}, \ldots$ be a fixed $\{0,1\}$-valued sequence. Define the infinite dimensional matrix $\left[A_{j l}\right]_{j, l=1,2 \ldots}$ by

$$
A_{j l}= \begin{cases}k_{0}^{x_{j}}\left(1-k_{0}\right)^{1-x_{j}} & \text { if } l=j \\ (-1)^{x_{j}+1} k_{1} & \text { if } l=j-1, j+1 \\ (-1)^{x_{j}+1} \frac{1}{k_{2}} & \text { if } l=j-2 \\ (-1)^{x_{j}+1} k_{2} & \text { if } l=j+2 \\ 0 \text { otherwise, } & \end{cases}
$$

and set $\Delta_{n}=\operatorname{det} A \mid\{1, \ldots, n\}$ for $n \geq 1, \Delta_{0}=1$, and $\Delta_{n}=0$ for $n<0$. Then $P_{n}^{f}\left(\left\{x_{(n)}\right\}\right)=\Delta_{n}$ for $n \geq 0$. Denote by $\left[\Delta_{n}\right]_{j}^{l}$ the determinant of the 
matrix that is obtained by deleting row $j$ and column $l$ from $A \mid\{1, \ldots, n\}$ $(n \geq 1, j, l=1, \ldots, n)$. Developing $\Delta_{n}$ along the last rows and columns, we obtain, for every $n \geq 3$,

$$
\begin{aligned}
\Delta_{n} & =\left(1-k_{0}\right)\left(\frac{k_{0}}{1-k_{0}}\right)^{x_{n}} \Delta_{n-1}-k_{1}^{2}(-1)^{x_{n}+x_{n-1}} \Delta_{n-2} \\
& -\left|k_{2}\right|^{2}\left(1-k_{0}\right)\left(\frac{k_{0}}{1-k_{0}}\right)^{x_{n-1}}(-1)^{x_{n}+x_{n-2}} \Delta_{n-3}+\left|k_{2}\right|^{4}(-1)^{x_{n}+x_{n-1}+x_{n-2}+x_{n-3}} \Delta_{n-4} \\
& +k_{1} k_{2}(-1)^{x_{n}+x_{n-2}}\left[\Delta_{n-1}\right]_{n-2}^{n-1}+k_{1} \bar{k}_{2}(-1)^{x_{n-2}+x_{n-3}}\left[\Delta_{n-1}\right]_{n-1}^{n-2}
\end{aligned}
$$

Furthermore, developing $\left[\Delta_{n-1}\right]_{n-2}^{n-1}$ and $\left[\Delta_{n-1}\right]_{n-1}^{n-2}$ along the last row and column, we obtain

$$
\begin{aligned}
& k_{1} k_{2}(-1)^{x_{n}+x_{n-2}}\left[\Delta_{n-1}\right]_{n-2}^{n-1}+k_{1} \bar{k}_{2}(-1)^{x_{n-2}+x_{n-3}}\left[\Delta_{n-1}\right]_{n-1}^{n-2} \\
& =-k_{1}^{2}\left(k_{2}+\bar{k}_{2}\right)(-1)^{x_{n}+x_{n-1}+x_{n-2}} \Delta_{n-3}-2 k_{1}^{2}\left|k_{2}\right|^{2}(-1)^{x_{n}+x_{n-1}+x_{n-2}+x_{n-3}} \Delta_{n-4} \\
& +\left|k_{2}\right|^{2}(-1)^{x_{n}+x_{n-1}}\left(k_{1} k_{2}(-1)^{x_{n-2}+x_{n-4}}\left[\Delta_{n-3}\right]_{n-4}^{n-3}+k_{1} \bar{k}_{2}(-1)^{x_{n-2}+x_{n-3}}\left[\Delta_{n-3}\right]_{n-3}^{n-4}\right)
\end{aligned}
$$

Substituting, we get

$$
\begin{aligned}
\Delta_{n} & =\left(1-k_{0}\right)\left(\frac{k_{0}}{1-k_{0}}\right)^{x_{n}} \Delta_{n-1}-k_{1}^{2}(-1)^{x_{n}+x_{n-1}} \Delta_{n-2} \\
& -\left|k_{2}\right|^{2}\left(1-k_{0}\right)\left(\frac{k_{0}}{1-k_{0}}\right)^{x_{n-1}}(-1)^{x_{n}+x_{n-2}} \Delta_{n-3}+\left|k_{2}\right|^{4}(-1)^{x_{n}+x_{n-1}+x_{n-2}+x_{n-3}} \Delta_{n-4} \\
& -k_{1}^{2}\left(k_{2}+\bar{k}_{2}\right)(-1)^{x_{n}+x_{n-1}+x_{n-2}} \Delta_{n-3}-2 k_{1}^{2}\left|k_{2}\right|^{2}(-1)^{x_{n}+x_{n-1}+x_{n-2}+x_{n-3}} \Delta_{n-4} \\
& +\left|k_{2}\right|^{2}(-1)^{x_{n}+x_{n-1}}\left(k_{1} k_{2}(-1)^{x_{n-2}+x_{n-4}}\left[\Delta_{n-3}\right]_{n-4}^{n-3}+k_{1} \bar{k}_{2}(-1)^{x_{n-2}+x_{n-3}}\left[\Delta_{n-3}\right]_{n-3}^{n-4}\right)
\end{aligned}
$$

Let us now compute $\Delta_{n}-\left|k_{2}\right|^{2}(-1)^{x_{n}+x_{n-1}} \Delta_{n-2}$, using equation (14) for $\Delta_{n}$ and equation (13) with $n-2$ in the place of $n$ for $\Delta_{n-2}$. Since the terms containing $\left[\Delta_{n-3}\right]_{n-4}^{n-3}$ and $\left[\Delta_{n-3}\right]_{n-3}^{n-4}$ cancel, we obtain

$$
\begin{aligned}
& \Delta_{n}-\left|k_{2}\right|^{2}(-1)^{x_{n}+x_{n-1}} \Delta_{n-2} \\
& =\left(1-k_{0}\right)\left(\frac{k_{0}}{1-k_{0}}\right)^{x_{n}} \Delta_{n-1}-k_{1}^{2}(-1)^{x_{n}+x_{n-1}} \Delta_{n-2} \\
& -\left|k_{2}\right|^{2}\left(1-k_{0}\right)\left(\frac{k_{0}}{1-k_{0}}\right)^{x_{n-1}}(-1)^{x_{n}+x_{n-2}} \Delta_{n-3}+\left|k_{2}\right|^{4}(-1)^{x_{n}+x_{n-1}+x_{n-2}+x_{n-3}} \Delta_{n-4} \\
& -k_{1}^{2}\left(k_{2}+\bar{k}_{2}\right)(-1)^{x_{n}+x_{n-1}+x_{n-2}} \Delta_{n-3}-2 k_{1}^{2}\left|k_{2}\right|^{2}(-1)^{x_{n}+x_{n-1}+x_{n-2}+x_{n-3}} \Delta_{n-4} \\
& -\left|k_{2}\right|^{2}(-1)^{x_{n}+x_{n-1}}\left(1-k_{0}\right)\left(\frac{k_{0}}{1-k_{0}}\right)^{x_{n-2}} \Delta_{n-3}+k_{1}^{2}\left|k_{2}\right|^{2}(-1)^{x_{n}+x_{n-1}+x_{n-2}+x_{n-3}} \Delta_{n-4} \\
& +\left|k_{2}\right|^{4}(-1)^{x_{n}+x_{n-1}+x_{n-2}+x_{n-4}}\left(1-k_{0}\right)\left(\frac{k_{0}}{1-k_{0}}\right)^{x_{n-3}} \Delta_{n-5} \\
& -\left|k_{2}\right|^{6}(-1)^{x_{n}+x_{n-1}+x_{n-2}+x_{n-3}+x_{n-4}+x_{n-5}} \Delta_{n-6}
\end{aligned}
$$

Equation (9) is obtained by rearranging the terms in the above equation. 
The following theorem gives recursive equations for the predictive probabilities of $P^{f}$.

Theorem 2. Let $P^{f}$ be a stationary, two-dependent determinantal probability measure on the cylinder sigma-algebra of $\{0,1\}^{Z}$ with $f$ as in (8), $k_{0} \in$ $(0,1), k_{1} \in \mathbb{R}$, and $k_{2} \in \mathbb{C}$. Then, for every $x \in\{0,1\}^{Z}$ and every $n \geq 3$

$$
\begin{aligned}
p_{n}^{f}\left(x_{(n-1)}\right) & =k_{0}-\sum_{j=2}^{6 \wedge n} a_{j} \prod_{u=1}^{j-1}\left(b\left(p_{n-u}^{f}\left(x_{(n-u-1)}\right) ; x_{n-u}\right)\right)^{-1} \\
& +|k|_{2}^{2} b\left(k_{0} ; x_{n-2}\right) \prod_{u=1}^{2}\left(b\left(p_{n-u}^{f}\left(x_{(n-u-1)}\right) ; x_{n-u}\right)\right)^{-1} \\
& -\sum_{j \in\{3,5\}, j \leq n} c_{j} b\left(k_{0} ; x_{n-j+2}\right) \prod_{u=1}^{j-1}\left(b\left(p_{n-u}^{f}\left(x_{(n-u-1)}\right) ; x_{n-u}\right)\right)^{-1}
\end{aligned}
$$

where $p_{1}^{f}\left(x_{(0)}\right):=P_{1}^{f}(\{1\}), a_{j}(2 \leq j \leq 6), b$ and $c_{j}(j=3,5)$ are defined as in (10), (11), and (12), respectively.

Proof. To prove (15), it is sufficient to compute

$$
p_{n}^{f}\left(x_{(n-1)}\right)=P_{n}^{f}\left(\left\{\left(x_{1}, \ldots, x_{n-1}, 1\right)\right\}\right) / P_{n-1}^{f}\left(\left\{\left(x_{1}, \ldots, x_{n-1}\right)\right\}\right)
$$

using (9), and notice that

$\frac{P_{n}^{f}\left(\left\{\left(x_{1}, \ldots, x_{n}\right)\right\}\right)}{P_{n-j}^{f}\left(\left\{\left(x_{1}, \ldots, x_{n-j}\right)\right\}\right)}=\prod_{u=0}^{j-1} p_{n-u-1}^{f}\left(x_{(n-u-2)}\right)^{x_{n-u-1}}\left(1-p_{n-u-1}^{f}\left(x_{(n-u-2}\right)\right)^{1-x_{n-u-1}}$

for every $n=1,2, \ldots$ and $j<n$.

The next corollary allows us to construct samples from $P_{n}^{f}$ starting from independent random variables. The proof is similar to that of Corollary 2.

Corollary 3. Let $k_{0} \in(0,1), k_{1} \in \mathbb{R}$ and $k_{2} \in \mathbb{C}$ be such that, for every $s \in \mathbb{T}$, $f(s):=\sum_{j=-2}^{2} k_{j} \exp (i 2 \pi j s) \in[0,1]$. Let $a_{j}(2 \leq j \leq 6), b$ and $c_{j}(j=3,5)$ be defined as in (10), (11) and (12), respectively. Furthermore, let $\left(Z_{n}\right)_{n \in \mathbb{N}}$ be a sequence of independent random variables defined on a probability space $(\Omega, \mathcal{F}, P)$ and uniformly distributed on $[0,1]$. 
If $X_{n}=1_{\left[0, W_{n}\right]}\left(Z_{n}\right)(n \geq 1), W_{1}=k_{0}, W_{2}=k_{0}+k_{1}^{2}\left(1-k_{0}\right)^{-1}\left(1-1 / k_{0}\right)^{X_{1}}$ and, for $n \geq 3$,

$$
\begin{aligned}
W_{n} & =k_{0}-\sum_{j=2}^{6 \wedge n} a_{j} \prod_{u=1}^{j-1} \frac{\left(1-1 / W_{n-u}\right)^{X_{n-u}}}{\left(1-W_{n-u}\right)} \\
& +\left|k_{2}\right|^{2} \frac{\left(1-1 / k_{0}\right)^{X_{n-2}}}{\left(1-k_{0}\right)} \prod_{u=1}^{2} \frac{\left(1-1 / W_{n-u}\right)^{X_{n-u}}}{\left(1-W_{n-u}\right)} \\
& -\sum_{j \in\{3,5\} j \leq n} c_{j} \frac{\left(1-1 / k_{0}\right)^{X_{n-j+2}}}{\left(1-k_{0}\right)} \prod_{u=1}^{j-1} \frac{\left(1-1 / W_{n-u}\right)^{X_{n-u}}}{\left(1-W_{n-u}\right)}
\end{aligned}
$$

then, for every $n \geq 1$, the probability distribution of $X_{(n)}$ coincides with $P_{n}^{f}$.

Equations (9) and (15) simplify when $k_{2}$ is real. Actually, recursive equations of lower degree hold in this case. It can be proved with techniques similar to the ones used in the proof of Theorem 2 that

$$
\begin{aligned}
p_{n}^{f}\left(\left\{x_{(n-1)}\right\}\right) & =k_{0}-k_{2}\left[1-b\left(k_{0} ; x_{n-1}\right)\left(b\left(p_{n-1}^{f}\left(x_{(n-2)}\right) ; x_{n-1}\right)\right)^{-1}\right] \\
& -\sum_{j=2}^{5 \wedge n} d_{j} \prod_{u=1}^{j-1}\left(b\left(p_{n-u}^{f}\left(x_{(n-u-1)}\right) ; x_{n-u}\right)\right)^{-1} \\
& +\sum_{j \in\{3,4\}, j \leq n} e_{j} b\left(k_{0} ; x_{n-j+2}\right) \prod_{u=1}^{j-1}\left(b\left(p_{n-u}^{f}\left(x_{(n-u-1)}\right) ; x_{n-u}\right)\right)^{-1}
\end{aligned}
$$

where $p_{1}^{f}\left(x_{(0)}\right):=P_{1}^{f}(\{1\}), b$ is defined as in (11),

$$
\begin{gathered}
d_{1}=k_{2} \quad d_{2}=-k_{1}^{2} \quad d_{3}=-k_{1}^{2} k_{2} \quad d_{4}=k_{2}^{4} \quad d_{5}=-k_{2}^{5}, \\
e_{j}=\left(-k_{2}\right)^{j-1} \quad(1 \leq j \leq 4) .
\end{gathered}
$$

Furthermore, one can construct a two-dependent $\operatorname{SDP} X_{(n)}$ with $k_{2} \in \mathbb{R}$ 
through $X_{n}=1_{\left[0, W_{n}\right]}\left(Z_{n}\right)(n \geq 1), W_{1}=k_{0}$,

$$
\begin{aligned}
W_{n} & =k_{0}-k_{2}\left[1-\frac{1-k_{0}}{1-W_{n-1}}\left(\frac{1-1 / W_{n-1}}{1-1 / k_{0}}\right)^{X_{n-1}}\right] \\
& -\sum_{j=2}^{5 \wedge n} d_{j} \prod_{u=1}^{j-1} \frac{\left(1-1 / W_{n-u}\right)^{X_{n-u}}}{1-W_{n-u}} \\
& +\sum_{j \in\{3,4\}, j \leq n} e_{j} \frac{1-k_{0}}{\left(1-1 / k_{0}\right)^{X_{n-j+2}}} \prod_{u=1}^{j-1} \frac{\left(1-1 / W_{n-u}\right)^{X_{n-u}}}{1-W_{n-u}},
\end{aligned}
$$

$(n \geq 2),\left(Z_{n}\right)_{n \in \mathbb{N}}$ independent and uniformly distributed on $[0,1]$.

The above results can be used either to simulate two-dependent SDP's sequentially with efficient algorithms or to explore the properties of the predictive distributions of $P^{f}$. The next example shows that the predictive probabilities of a non-degenerate two-dependent SDP converge nowhere a.s.

Example 3. Let $\left(X_{n}\right)_{n \in Z}$ be an SDP with $f$ as in (8), $k_{0} \in(0,1), k_{1}, k_{2} \in \mathbb{R}$. We will prove that $0<\liminf _{n \rightarrow \infty} p_{n}^{f}\left(X_{(n-1)}\right)<\limsup _{n \rightarrow \infty} p_{n}^{f}\left(X_{(n-1)}\right)<1$ a.s.

Let us first show that $\liminf _{n \rightarrow \infty} p_{n}^{f}\left(X_{(n-1)}\right)>0$ a.s. Since for every $x_{(n-1)} \in\{0,1\}^{n-1}, p_{n}^{f}\left(x_{(n-1)}\right) \leq p_{n}^{f}\left(1_{(n-1)}\right)$ (see Lyons, 2003), it holds $\liminf \inf _{n \rightarrow \infty} p_{n}^{f}\left(X_{(n-1)}\right) \geq \liminf _{n \rightarrow \infty} p_{n}^{f}\left(1_{(n-1)}\right)$.

On the other hand, $\left(p_{n}^{f}\left(1_{(n-1)}\right)\right)_{n \geq 2}$ is a decreasing sequence converging to $p:=\exp \int_{\mathbb{T}} \log f d \lambda$ (see Lyons, 2003). To show that $p>0$ without evaluating the integral, we can use (16). In fact $p$ must satisfy $p^{5}=\left(k_{0}-k_{1}\right) p^{4}+\left(k_{0} k_{2}-\right.$ $\left.k_{1}^{2}\right) p^{3}-k_{2}\left(k_{0} k_{2}-k_{1}^{2}\right) p^{2}-k_{2}^{3}\left(k_{0}-k_{2}\right) p+k_{2}^{5}$ and $p=0$ is not a solution of this equation. By duality, $q:=\lim _{n \rightarrow \infty} p_{n}^{f}\left(0_{(n-1)}\right)<1$.

Let us now prove that $\liminf _{n \rightarrow \infty} p_{n}^{f}\left(X_{(n-1)}\right)<\lim \sup _{n \rightarrow \infty} p_{n}^{f}\left(X_{(n-1)}\right)$ a.s. Let $M=\cap_{n=1}^{\infty} \cup_{k=n}^{\infty}\left\{x \in\{0,1\}^{Z}: x_{k-1}=1, \ldots, x_{k-5}=1\right\}$ and let $N=\cap_{n=1}^{\infty} \cup_{k=n}^{\infty}\left\{x \in\{0,1\}^{Z}: x_{k-1}=1, \ldots, x_{k-4}=1, x_{k-5}=0\right)$. Since $P^{f}$ is a Bernoulli shift (see Theorem 3.1 in Lyons and Steif, 2003), it is ergodic. Hence $P^{f}(M)$ and $P^{f}(N)$ are either equal to zero or equal to one. On the other hand, $P^{f}$ has full support (see Theorem 4.2 in Lyons and Steif, 2003). Hence $P^{f}(M)=P^{f}(N)=1$. Let now $Q=\{x \in$ $\left.\{0,1\}^{\mathbb{Z}}: \liminf _{n \rightarrow \infty} p_{n}^{f}\left(x_{(n-1)}\right)=\limsup _{n \rightarrow \infty} p_{n}^{f}\left(x_{(n-1)}\right)\right\}$. Then $P^{f}(Q \cap$ $M \cap N)=P^{f}(Q)$. Suppose that there exists $x \in M \cap N \cap Q$ and let 
$p(x)=\lim _{n \rightarrow \infty} p_{n}^{f}\left(x_{(n-1)}\right)$. Then, by (16),

$$
\begin{aligned}
p(x) & =k_{0}-k_{2}+\frac{k_{0} k_{2}-k_{1}^{2}}{p(x)}-k_{2} \frac{k_{0} k_{2}-k_{1}^{2}}{p(x)^{2}}-k_{2}^{3} \frac{k_{0}-k_{2}}{p(x) 3^{3}}+\frac{k_{2}^{5}}{p(x)^{4}} \\
& =k_{0}-k_{2}+\frac{k_{0} k_{2}-k_{1}^{2}}{p(x)}-k_{2} \frac{k_{0} k_{2}-k_{1}^{2}}{p(x)^{2}}-k_{2}^{3} \frac{k_{0}-k_{2}}{p(x)^{3}}-\frac{k_{2}^{3}(x)^{3}(1-p(x))^{2}}{p} .
\end{aligned}
$$

Simplifying, we find $\frac{k_{2}^{5}}{p(x)^{4}}=\frac{k_{2}^{5}}{p(x)^{3}(p(x)-1)}$ which leads to a contradiction since $k_{2} \neq 0$. We deduce that $M \cap N \cap Q=\emptyset$ and, therefore $P^{f}(Q)=0$.

We close by noting that the problem is still open whether fixed order recursive equations hold for $m$-dependent SDP's when $m>2$.

Acknowledgements. The authors highly appreciate the reviewer and the Associate Editor for their insightful comments and useful suggestions that have helped us to greatly improve this manuscript.

\section{References}

Borodin, A., Diaconis, P. And Fulman, J. (2009). On adding a list of numbers (and other one-dependent determinantal processes) arXiv:0904.3740.

Broman, E. I. (2005). One-dependent trigonometric determinantal processes are two-block-factors. Ann. Probab. 33, 601-609.

Cifarelli, D. M. And Fortini, S. (2005). A short note on one-dependent trigonometric determinantal probability measures. Technical report, Istituto di Metodi Quantitativi, Università Bocconi.

Lyons, S. (2003). Determinantal probability measures. Publ. Math. Inst. Hautes Études Sci. 98, 167-212.

Lyons, S. And Steif, J. (2003). Stationary determinantal processes: Phase transitions, Bernoullicity, entropy and domination. Duke Math. J. 120, 515-575.

Shirai, T. And Takahashi, Y. (2003). Random point fields associated with certain Fredholm determinants II: fermion shifts and their ergodic and Gibbs properties. Ann. Probab. 31, 1533-1564. 